\title{
Abordagem da linguagem na doença de Alzheimer: estudo sobre panorama brasileiro de pesquisas
}

\author{
Nathália Luiz Freitas*
}

\begin{abstract}
Resumo: A Doença de Alzheimer (DA) é caracterizada por prejuízo irreversível da memória e por alterações cognitivas e comportamentais que interferem nas práticas sociais cotidianas. $\mathrm{O}$ comprometimento da linguagem na DA ocorre nos três estágios da neurodegenerescência, se traduzindo, na forma leve, por alterações nos aspectos semântico-lexicais-pragmáticos, na forma moderada, por alterações fonológicas, sintáticas e morfológicas, e, na forma severa, por prejuízo em todas as habilidades linguísticas, levando o sujeito, muitas vezes, ao mutismo. Considerando que a linguagem é uma das funções cognitivas significativamente afetadas na DA, este artigo pretende investigar as abordagens acadêmico-científicas brasileiras sobre a linguagem em tal doença. Para tanto, foi realizado levantamento bibliográfico sistemático nos portais de periódicos Capes e Scielo, além do Google Acadêmico, utilizando-se como termos de busca os vocábulos linguagem e Alzheimer em conjunto. Os estudos encontrados foram analisados e classificados conforme pertencimento à área do conhecimento, perspectiva subjacente de linguagem e teoria lingüística empregada. O levantamento bibliográfico realizado indica baixo número de estudos sobre linguagem na DA no Brasil, predominância da Linguística em tais pesquisas, prevalência da perspectiva estrutural de linguagem e escasso emprego de teorias linguísticas nas análises dos dados.
\end{abstract}

Palavras-chave: Doença de Alzheimer; Linguagem; Linguística.

Abstract: Alzheimer's Disease (AD) is characterized by irreversible loss of memory and by cognitive and behavioral changes that interfere in everyday social practices. The language impairment in $\mathrm{AD}$ occurs in three stages of neurodegeneration: in a mild form, by changes in aspects of lexical-semantic-pragmatic; in a moderate way, by phonological, morphological and syntactic alterations; and in a severe form, by loss in all language skills, usually leading the subject to silence. Considering that language is a cognitive function significantly affected in $\mathrm{AD}$, this paper aims to investigate the Brazilian academic-scientific approaches on language in such a disease. Systematic literature review was conducted in the databases of CAPES and Scielo, and at Google Scholar, using as search terms language and Alzheimer together. The studies found were analyzed and classified as belonging to specific areas of knowledge, underlying the perspective of using language and linguistic theory. The bibliographical survey indicates a low number of studies on language in $\mathrm{AD}$ in Brazil, the predominance of Linguistics in such surveys, the prevalence of structural perspective of language and sparse use of linguistic theories in the analysis of the data.

Keywords: Alzheimer's disease; Language; Linguistics.

\footnotetext{
* Mestranda em Estudos da Linguagem, UFOP. Professora do IFSULDEMINAS, Poços de Caldas.
} 


\section{Considerações Iniciais}

\subsection{Linguagem na Doença de Alzheimer}

Descrita pelo alemão Aloïs Alzheimer, em 1907, a Doença de Alzheimer (doravante, DA) se traduz por alterações cognitivas e comportamentais que constituem uma síndrome demencial adquirida ${ }^{1}$. A DA tem como característica o declínio de memória juntamente com outro déficit cognitivo, como de linguagem, habilidades visuoespaciais ou de controle executivo, combinação suficiente para interferir nas atividades sociais do sujeito (APRAHAMIAN et. al., 2008). Trata-se do tipo mais comum entre as demências, atingindo mais da metade das que são diagnosticadas. Embora sua etiologia não seja totalmente conhecida, entre suas causas podemos encontrar fatores genéticos, fatores de risco (antecedentes mórbidos como traumatismos cranianos com perda de consciência, arteriosclerose e diabetes) e depressões tardias, não tratadas (MORATO, 2008).

Segundo Morato (2008), na área neurocognitiva, são descritas três fases de evolução da doença: i) a forma leve, em que os problemas de memória são constantes, havendo, no que tange à linguagem, relativa preservação dos aspectos fonológicosintáticos e alterações nas habilidades semântico-lexicais-pragmáticas, as quais são sutis e, por isso, frequentemente, passam despercebidas; ii) a forma moderada, na qual os problemas mnésicos já chegam a ser incapacitantes, com desorientação têmporoespacial e linguística. Nessa fase, os problemas de linguagem, ainda não claramente observáveis na fase anterior, passariam a ser frequentes e prontamente perceptíveis. Somados a eles, os problemas práxicos e gnósicos configurariam o que é chamado por muitos autores de síndrome afásico-apráxico-agnósica; iii) $\mathrm{E}$ a forma severa, durante a qual a memória se encontra gravemente alterada e a linguagem apresenta-se significativamente prejudicada. No estágio mais avançado da doença, as habilidades linguísticas gerais ficam sensivelmente comprometidas, podendo o sujeito acometido pela DA chegar ao mutismo.

De forma geral, o estudo da DA, sob as diversas esferas do conhecimento, se justifica, no que se refere ao cenário acadêmico brasileiro, em razão do significativo

\footnotetext{
${ }^{1}$ As síndromes demenciais consistem em morbidades, em sua maioria, degenerativas e progressivas, as quais implicam significativos transtornos mental, físico e psicológico para o indivíduo acometido. (APRAHAMIAN et. al., 2008).
} 
aumento da população idosa, e consequente elevação da prevalência de doenças relacionadas à senilidade, em especial as demências, ocorrido nos últimos anos no Brasil. Conforme Aprahamian e colaboradores (2009), que se baseiam em dados do IBGE - Instituto Brasileiro de Geografia e Estatística - referentes ao ano de 2007, a população brasileira acima de 60 anos cresce a cada ano, chegando aos dias atuais a cerca de 15 milhões de pessoas, sendo estimada a aumentar em 15\% no ano de 2020. De acordo com os referidos autores, a DA é a mais prevalente entre as demências senis $50 \%$-, prevalência esta que aumenta progressivamente com o envelhecimento. A partir dos 65 anos, o número de acometidos dobra a cada cinco anos.

Considerando que a linguagem, e principalmente a língua - tanto em seu domínio estrutural (fonológico, morfológico, sintático, morfossintático) quanto em sua esfera interacional/social (semântica, pragmática) -, é uma das funções cognitivas significativamente afetadas na DA (NOGUCHI, 1998), e levando-se em conta ainda que a referida demência provoca elevado impacto biopsicossocial na sociedade contemporânea, conforme exposto acima, torna-se relevante a realização de um estudo que, em linhas gerais, vise a investigar as abordagens acadêmico-científicas brasileiras sobre a linguagem na DA, de modo a averiguar como tal capacidade cognitiva vem sendo compreendida e tratada. Tal investigação pode contribuir para a compreensão dos pressupostos que embasam a testagem diagnóstica, assim como para o entendimento da natureza das estratégias de reabilitação em DA que vêm sendo empregadas no que se refere ao comprometimento das habilidades linguísticas. $\mathrm{O}$ presente estudo pode ainda, para fins teóricos, mostrar qual a participação de estudiosos do âmbito da Linguística e a utilização de teorias concernentes à Ciência da Linguagem na análise das alterações linguísticas em sujeitos acometidos pela neurodegenerescência em pauta.

No tópico a seguir são apresentados os objetivos desta investigação. Nas seções seguintes, estão, respectivamente, descritos a metodologia empregada, assim como os resultados obtidos nesta pesquisa. Nas Considerações Finais são tecidos comentários sobre os resultados encontrados.

\section{Objetivos}

Diante do contexto exposto, objetiva-se: 
1) Averiguar a área do conhecimento (Medicina, Psicologia, Fonoaudiologia, Linguística etc.) em que cada um dos estudos sobre Linguagem e Doença de Alzheimer empreendidos no cenário brasileiro se insere;

2) Investigar as perspectivas de linguagem que subjazem aos referidos estudos;

3) Identificar, entre as perspectivas de linguagem apuradas, a utilização de teorias linguísticas no que tange às análises dos dados linguísticos presentes nos estudos encontrados.

\section{Metodologia}

Para alcançar os objetivos propostos, foi realizado levantamento bibliográfico sistemático, o qual se caracteriza por pesquisa exaustiva em determinado banco de dados, com vistas a averiguar todas as ocorrências do fenômeno em estudo. Optou-se pela utilização dos portais de periódicos Capes - onde estão indexados periódicos de Qualis elevados, além de teses e dissertações produzidas em instituições de reconhecimento, e do qual serão retirados apenas os trabalhos desenvolvidos no Brasil e Scielo - portal cujas revistas, brasileiras, gozam de reconhecido prestígio -, além do Google Acadêmico, site de busca em que, além de papers, podem ser encontradas dissertações e teses indexadas por endereços eletrônicos de universidades. Foram utilizados como termos de busca os vocábulos linguagem e Alzheimer em conjunto, baseando-se no pressuposto de que as palavras-chave presentes nos trabalhos acadêmicos têm a função de sintetizar os temas neles abordados.

Após a etapa de busca, foi feita uma triagem dos trabalhos, com vistas a descartar aqueles que não versam sobre a linguagem na Doença de Alzheimer. Posteriormente, os estudos encontrados sobre a temática em pauta foram analisados de forma a serem classificados conforme pertencimento à área do conhecimento, perspectiva subjacente de linguagem e teoria linguística empregada. As categorias específicas de cada tipo de classificação foram sendo estabelecidas à medida que os estudos foram analisados, conforme pode ser observado na seção destinada aos resultados, logo abaixo.

\section{Resultados}


Por meio de busca realizada nos referidos portais, foram encontrados 39 estudos. Desse total, 33 versam especificamente sobre a linguagem na DA, sendo os demais concernentes a outros aspectos como, por exemplo, critérios diagnósticos e epidemiologia, de forma que, em tais trabalhos, a referida competência tem papel secundário. Esses 6 estudos foram, portanto, excluídos das análises. Dos 33 trabalhos restantes, 3 são publicações em anais de eventos referentes a recortes de dissertações averiguadas pelo presente estudo, sendo, por isso, de igual forma, excluídos. Na tabela 1, apresentada a seguir, são sucintamente indicados os 30 estudos encontrados sobre linguagem na DA e levados em consideração neste artigo.

Tabela 1: Dados bibliográficos e principais objetivos dos estudos encontrados sobre linguagem na Doença de Alzheimer.

\begin{tabular}{l|l}
\hline \multicolumn{1}{c}{ Título, Autores e Ano do estudo } & \multicolumn{1}{|c}{ Principais Objetivos } \\
\hline $\begin{array}{l}\text { A linguagem na doença de Alzheimer: } \\
\text { considerações sobre um modelo de } \\
\text { funcionamento lingüístico-cognitivo, } \\
\text { Noguchi, 1997. }\end{array}$ & $\begin{array}{l}\text { Investigar atureza do } \\
\text { comprometimento semântico presente na } \\
\text { DA, bem como uma possível relação } \\
\text { existente entre linguagem e percepção } \\
\text { visual. }\end{array}$ \\
\hline $\begin{array}{l}\text { Memória e compreensão da linguagem no } \\
\text { envelhecimento, Parente et. al., 1999. }\end{array}$ & $\begin{array}{l}\text { Focalizar os efeitos etários na memória } \\
\text { verbal de curto e de longo prazo. }\end{array}$ \\
\hline $\begin{array}{l}\text { Caracterização dos distúrbios da escrita na } \\
\text { doença de Alzheimer, Carthery, 2000. }\end{array}$ & $\begin{array}{l}\text { Caracterizar os distúrbios de escrita na } \\
\text { DA, analisando os tipos de agrafia de } \\
\text { acordo com a abordagem cognitiva e } \\
\text { verificando os quadros mais frequentes. }\end{array}$ \\
\hline $\begin{array}{l}\text { Análise das habilidades fonológicas no } \\
\text { envelhecimento normal e na doença de }\end{array}$ & $\begin{array}{l}\text { Comparar os índices de consciência } \\
\text { fonológica entre idosos com DA e idosos } \\
\text { Alzheimer, Carvalho \& Assencio-Ferreira, } \\
\text { sem DA. }\end{array}$ \\
$\begin{array}{l}\text { O02. } \\
\text { tipo Alzheimer, Rodrigues, 2003. }\end{array}$ & $\begin{array}{l}\text { Revisar a literatura sobre o processamento } \\
\text { sintático na DA. }\end{array}$ \\
\hline $\begin{array}{l}\text { Linguagem e cognição na Doença de } \\
\text { Alzheimer, Mansur et. al., 2005. }\end{array}$ & $\begin{array}{l}\text { Revisar a literatura recente sobre a } \\
\text { linguagem na DA, e, especificamente, } \\
\text { estudos com população brasileira. }\end{array}$ \\
\hline $\begin{array}{l}\text { Alterações de linguagem nas fases iniciais } \\
\text { da doença de Alzheimer, Ortiz \& } \\
\text { Bertolucci, 2005. }\end{array}$ & $\begin{array}{l}\text { Verificar a presença de alterações de } \\
\text { linguagem em pacientes com DA em fase } \\
\text { inicial. }\end{array}$ \\
\hline $\begin{array}{l}\text { A nomeação de figuras e o acesso lexical } \\
\text { na demência de Alzheimer: um estudo de } \\
\text { caso, Oliveira \& Stivanin, 2005. }\end{array}$ & $\begin{array}{l}\text { Avaliar a memória semântica em um } \\
\text { paciente com DA através de um teste de } \\
\text { nomeação de figuras e acesso lexical. }\end{array}$ \\
\hline $\begin{array}{l}\text { Perfil discursivo e interativo de pessoas } \\
\text { com Doença de Alzheimer, Brandão, } \\
\text { 2005. }\end{array}$ & $\begin{array}{l}\text { Investigar a coerência e os déficits no } \\
\text { manejo do conhecimento de pessoas com } \\
\text { DA em três tarefas de discurso, } \\
\text { comparando as relações dessas } \\
\text { características com habilidades cognitivas. }\end{array}$ \\
\hline
\end{tabular}


O discurso narrativo na Doença de Alzheimer e na Demência FrontoTemporal, Samara, 2005.

Avaliação funcional das habilidades de comunicação ASHA FACS para a população com doença de Alzheimer, Carvalho, 2006.

A linguagem em sujeitos com Demência de Alzheimer, sob a ótica de uma concepção enunciativo-discursiva, Gomes, 2007.

Considerações sobre a relação linguagemmemória, à luz da análise lingüística dos enunciados de sujeitos com diagnóstico de demência de Alzheimer, Beilke \& Novaes-Pinto, 2007.

Influência do hipertexto na compreensão textual de pacientes com demência de Alzheimer leve e moderada, Rinaldi, 2007.

Turnos e atos de fala do interlocutor de pessoas com Doença de Alzheimer, Brandão et. al., 2008.

$\begin{aligned} & \text { Investigar a habilidade } \\ & \text { narrativa e discursivo- } \\ & \text { neuropsicológicos. }\end{aligned}$
Validar a escala de avaliação ASHA

FACS para uso na população brasileira.

Atentar para a importância em se avaliar o componente pragmático da linguagem de sujeitos com DA.

Refletir sobre a relação entre linguagem e memória, a partir da análise da linguagem de sujeitos com DA.

Verificar se o hipertexto facilita o reconto de idosos com DA.

Investigar a frequência de turnos e atos de fala do interlocutor na interação com participantes nos estágios de declínio cognitivo moderado e moderado-severo da doença de Alzheimer (DA) em diferentes tarefas discursivas.

Linguagem, interação e cognição na doença de Alzheimer, Cruz, 2008.

A compreensão de leitura textual como um instrumento de diagnóstico de pacientes com demência de Alzheimer leve e moderada, Rinaldi et.al., 2008.

O caráter sociocognitivo da metaforicidade: contribuições do estudo do tratamento de expressões formulaicas por pessoas com afasia e com Doença de Alzheimer, Morato, 2008.

A desintegração de tempo lingüístico em Alzheimer, Martins \& Novaes, 2008.

Avaliação de linguagem na Demência de Alzheimer, Novaes-Pinto \& Beilke, 2008.

Aspectos pragmáticos na linguagem de um sujeito com diagnóstico de Demência de Alzheimer, Batista et. al., 2009.

Linguagem e memória na doença de Alzheimer em fase moderada, Azevedo et. al., 2009.
Investigar, a partir de uma perspectiva sócio-cognitiva, a linguagem em interação na DA.

Verificar se, desde as etapas iniciais, os pacientes com DA leve e moderada se mostram sensíveis à tarefa de compreensão de leitura textual.

Analisar aspectos linguísticosinteracionais da interpretação de expressões formulaicas por pessoas com afasia e com DA em fase inicial.

Elucidar a natureza dos déficits linguísticos na DA.

Discutir criticamente a avaliação da linguagem na DA.

Averiguar as estratégias utilizadas por um sujeito com DA em fase inicial durante situações dialógicas.

Analisar alterações de memória e de linguagem em um paciente com DA na fase moderada, verificando a interferência 


\begin{tabular}{|c|c|}
\hline & $\begin{array}{l}\text { das variáveis idade e sexo nessas } \\
\text { competências cognitivas. }\end{array}$ \\
\hline $\begin{array}{l}\text { Comparação das habi } \\
\text { comunicação na depressão de } \\
\text { e doença de Alzheimer, Nova }\end{array}$ & $\begin{array}{l}\text { Verificar o desempenho de idosos sadios, } \\
\text { pacientes deprimidos e pacientes } \\
\text { portadores de DA na fase inicial em } \\
\text { habilidades de comunicação, de modo a } \\
\text { comparar os perfis de tais grupos. }\end{array}$ \\
\hline $\begin{array}{l}\text { Interação e linguagem: Notas } \\
\text { de idosos em situações cc } \\
\text { Sampaio et. al., } 2009 .\end{array}$ & $\begin{array}{l}\text { Abordar, de forma sucinta, o } \\
\text { funcionamento da linguagem de um } \\
\text { sujeito com a Doença de Alzheimer e um } \\
\text { sujeito com afasia. }\end{array}$ \\
\hline $\begin{array}{l}\text { Habilidades lingüísticas de pacientes com } \\
\text { demência vascular: estudo comparativo } \\
\text { com a doença de Alzheimer, Freitas, } \\
2010 \text {. }\end{array}$ & $\begin{array}{l}\text { Descrever as habilidades linguísticas de } \\
\text { pacientes com DV em fase leve e } \\
\text { compará-las com as habilidades } \\
\text { linguísticas de pacientes com DA e idosos } \\
\text { cognitivamente normais. }\end{array}$ \\
\hline $\begin{array}{llr}0 & \text { em } & \mathrm{Al} \\
\text { eito e memória }\end{array}$ & $\begin{array}{l}\text { Investigar a existência e a origem do } \\
\text { déficit na expressão linguística de tempo } \\
\text { em sujeitos com DA. }\end{array}$ \\
\hline $\begin{array}{l}\text { gias comunicativas de pessoas com } \\
\text { de Alzheimer, Brandão et. al., }\end{array}$ & $\begin{array}{l}\text { tigar estratégias compens } \\
\text { em na comunicação de sl }\end{array}$ \\
\hline $\begin{array}{l}\text { A narrativa na demência de Alzheimer: } \\
\text { reorganização da linguagem e das } \\
\text { "memórias" por meio de práticas } \\
\text { dialógicas, Beilke \& Novaes-Pinto, } 2010 .\end{array}$ & $\begin{array}{l}\text { Analisar aspectos do funcionamento da } \\
\text { linguagem de sujeitos com DA, por meio } \\
\text { de suas narrativas. }\end{array}$ \\
\hline $\begin{array}{lll}\text { Alguns aspectos relacionados } & \text { ao } \\
\text { funcionamento da linguagem } & \text { no } \\
\text { envelhecimento, Sampaio, 2010. } & \end{array}$ & n sujeito com DA. \\
\hline $\begin{array}{l}\text { Manifestações da ar } \\
\text { doença de Alzheimer, }\end{array}$ & $\begin{array}{l}\text { Identificar as manif } \\
\text { pacientes com doen }\end{array}$ \\
\hline
\end{tabular}

Os 30 trabalhos encontrados dividem-se em 6 teses, 4 dissertações e 20 artigos. Conforme pode ser observado, embora os objetivos dos estudos sejam bastante variados, todos eles focalizam a língua(gem) como componente cognitivo alterado em razão da DA. Além disso, cabe ressaltar o quão recente é o trabalho mais antigo publicado no cenário brasileiro, entre os encontrados nessa revisão, datando de 1997. Outro fator que merece destaque é o número relativamente baixo de estudos sobre a linguagem na DA, se comparado, por exemplo, com a quantidade de estudos realizados acerca da linguagem na afasia, patologia de linguagem por excelência. Na tabela 2, 
listada abaixo, são apresentadas as áreas do conhecimento a que pertencem os trabalhos encontrados.

Tabela 2: Número de trabalhos sobre linguagem na Doença de Alzheimer conforme área do conhecimento.

\begin{tabular}{c|c}
\hline Área do Conhecimento & Número de Estudos \\
\hline Linguística & 12 \\
\hline Psicologia & 8 \\
\hline Medicina & 5 \\
\hline Fonoauadiologia & 4 \\
\hline Neurociências & 1 \\
\hline Total & 30 \\
\hline
\end{tabular}

Como pode ser observado na tabela 2, entre as áreas do conhecimento em que os estudos se inserem, predomina a Linguística, seguida pela Psicologia e pela Medicina, o que indica que a ciência cujo objeto de estudo é a língua(gem) é a que mais se ocupa da linguagem na DA, de modo a apontar também representativa participação de áreas do conhecimento que tradicionalmente tratam da linguagem em tal neurodegenerescência, ou seja, a Psicologia e a Medicina. No que tange aos estudos encontrados que estão no âmbito da Linguística, vale salientar que cerca de metade deles é filiada à Neurolinguística Discursiva, perspectiva teórico-metodológica que concebe língua, discurso, cérebro e mente como construtos humanos que se relacionam (COUDRY, 2008), tendo nas patologias que afetam a linguagem excelente expediente de pesquisa. Todos os trabalhos encontrados cujo arcabouço teórico-metodológico é a Neurolinguística Discursiva buscam privilegiar uma abordagem dialógica de linguagem, enfatizando o papel da interação na busca pela compensação dos déficits linguísticos na DA.

No que diz respeito às perspectivas de linguagem que subjazem aos estudos em análise, foram encontradas a estrutural e a enunciativo-discursiva. A perspectiva estrutural concebe a língua/linguagem de forma organicista, ou seja, como um sistema autônomo, e imanente em que as significações são pré-estabelecidas. O Estruturalismo Linguístico (SAUSSURE, 1986), bem como o Gerativismo (CHOMSKY, 1980) podem ser consideradas bases epistemológicas para tal abordagem. A perspectiva enunciativodiscursiva, pautada nas ideias bakhitinianas (BAKHTIN, 2000), entende a língua/linguagem como um construto sócio-histórico-cognitivo-cultural ancorado nas práticas dialógicas. Diferentemente da concepção estrutural, a concepção enunciativo- 
discursiva assente que os sentidos são construídos entre os interlocutores através da interação. Na tabela 3, abaixo, está o número de trabalhos conforme perspectiva de linguagem subjacente.

Tabela 3: Número de trabalhos encontrados sobre linguagem na Doença de Alzheimer de acordo com a perspectiva de linguagem subjacente.

\begin{tabular}{c|c}
\hline Perspectiva de Linguagem & Número de Trabalhos \\
\hline Estrutural & 16 \\
\hline Enunciativo-discursiva & 14 \\
\hline Total & 30 \\
\hline
\end{tabular}

Como pode ser visualizado, predomina, nos estudos sobre linguagem na DA, a perspectiva estrutural de linguagem. Faz-se necessário enfatizar que, desses 16 trabalhos de perspectiva estrutural, 3 são provenientes do âmbito da Linguística e os demais da Fonoaudiologia, da Psicologia e da Medicina, áreas do saber em que, tradicionalmente, está subjacente tal abordagem.

No que se refere ao emprego de teorias linguísticas na análise de dados, apenas 1 dos 30 estudos o faz. Trata-se de um trabalho da subárea de Neurolinguística Discursiva que utiliza as Máximas Conversacionais de Grice (1970). Dos 29 estudos restantes, 10 assumem como arcabouço metodológico de análise uma "abordagem qualitativa", 15 utilizam testes metalinguísticos, os quais, por sua vez, são analisados por meio das faixas de interpretação propiciadas pelos seus escores e 4 consistem em revisão de literatura. Cabe ressaltar que os trabalhos que empregam como instrumento de análise unicamente a testagem metalinguística concebem a língua/linguagem de maneira estrutural, desconsiderando o dialogismo inerente à comunicação humana. Contudo, esses trabalhos propiciam o entendimento sobre o comprometimento de determinados aspectos linguísticos basilares à comunicação humana que uma abordagem dialógica não possui ferramentas para investigar.

Abaixo são feitas considerações sobre os resultados acima descritos, a fim de propiciar reflexões tangentes a eles.

\section{Considerações Finais}


Os resultados oriundos do presente trabalho de revisão bibliográfica sistemática propiciam a reflexão acerca de, ao menos, quatro problemáticas. A primeira delas diz respeito ao baixo número de trabalhos sobre linguagem na Doença de Alzheimer publicados no Brasil, de acordo com o que foi encontrado nos já referidos portais. A quantidade de 30 estudos, entre artigos, dissertações e teses, mostra que essa é uma temática ainda pouco estudada, o que indica a necessidade de elevação de tal número, haja vista o aumento da população idosa brasileira e os dados epidemiológicos sobre a DA, apontados no início deste artigo. Além disso, os poucos trabalhos desenvolvidos estão concentrados em determinados grupos de pesquisa. Isso pode evidenciar o emprego de arcabouços teórico- metodológicos semelhantes, aspecto passível de contribuir com o predomínio de certas perspectivas em detrimento da diversidade de enfoques desejada pela Ciência.

A segunda questão refere-se à atuação significativa da Linguística no empreendimento de estudos sobre a linguagem na DA, uma vez que, dos 30 trabalhos encontrados, 12 - quase a metade deles - pertencem a tal área do conhecimento. Esse fato indica certa expressividade da Ciência da Linguagem no que tange às pesquisas sobre as neurodegenerescências, além de responder a uma importante crítica feita pelo linguista Roman Jakobson (1956), que, ao discorrer sobre a afasia, atenta para a necessidade de os cientistas da linguagem se ocuparem do estudo de patologias que envolvem a linguagem, fornecendo a elas uma abordagem, de fato, linguística.

Outro aspecto a ser refletido concerne à predominância da perspectiva estrutural de linguagem nos estudos sobre linguagem na DA. Várias são as críticas aos estudos que empregam testes neuropsicológicos como única forma de avaliar a linguagem na DA. Tais críticas podem ser resumidas na adoção de uma visão de língua estruturalista, por meio da utilização de tarefas metalinguísticas, em detrimento de uma abordagem dialógica que considera o funcionamento da linguagem, através do sócio-interacionismo (NOGUCHI, 1998; DAMASCENO, 1999; CRUZ, 2004; BEILKE \& NOVAES-PINTO, 2008). Assim, a apuração das perspectivas de linguagem subjacentes aos estudos em análise reforça o referido posicionamento, mostrando que a maioria dos estudos acerca da linguagem na DA empreitada no Brasil ainda tem como base uma perspectiva reducionista de língua/linguagem. Compreender a língua/linguagem apenas dessa maneira pode tanto dificultar o diagnóstico precoce da DA, quanto comprometer as atividades terapêuticas desenvolvidas. Isso em decorrência de, como salientam Beilke \& 
Novaes-Pinto (2007), as alterações de linguagem em estágio inicial consistirem em evidências sutis, não sendo notadas nas primeiras entrevistas, nem detectadas nos testes neuropsicológicos. Além disso, ao não se privilegiar a dimensão dialógica em acompanhamentos de reabilitação, nega-se ao sujeito acometido a oportunidade de recompensar seus déficits com estratégias interlocutivas.

É necessário assinalar, entretanto, que estudos do tipo estruturalista foram fundamentais para o desenvolvimento da abordagem dialógica, haja vista que possibilitaram a compreensão de diversos processos linguísticos alterados quando do acometimento do sujeito pela DA. Além disso, o emprego de testagem metalinguística tende a evidenciar prejuízos nas habilidades morfológicas, fonológicas, sintáticas e, dependendo do teste, semânticas, elucidação esta importante na investigação sobre as alterações de linguagem sofridas pelo paciente diagnosticado com a DA. Dessa maneira, o que se coloca na reflexão em pauta não é o abandono da perspectiva estruturalista com relação à linguagem na DA, mas, sim, quando possível, a junção do olhar estrutural com o dialógico, ou então, a definição de limites, por parte das pesquisas estruturalistas, na dimensão do que é considerado língua(gem).

O último fator a ser apontado relaciona-se à utilização de teorias linguísticas nas análises de dados fornecidos por sujeitos com DA nos estudos sobre linguagem no cenário acadêmico brasileiro. Tal emprego é, como exposto, quase nulo. Tal fato pode ser explicado em razão do elevado número de trabalhos que utilizam somente os escores dos testes metalinguísticos aplicados para avaliar a linguagem, e em virtude do uso da chamada "abordagem qualitativa" nas análises de dados coletados por meio de entrevistas e de narrativas. Não se pretende aqui questionar o mérito científico das análises pautadas na referida abordagem, mas sim atentar para a possibilidade de se alcançar maior robustez analítica através do emprego de teorias linguísticas, principalmente as pragmático-cognitivas, largamente utilizadas pelos Estudos Linguísticos em pesquisas sobre outros fenômenos de linguagem.

Tecidos tais comentários, fica evidente a necessidade urgente em se empreenderem mais estudos sobre a linguagem na Doença de Alzheimer. Ressalta-se a importância de tais estudos considerarem a língua/linguagem também como uma atividade criadora de natureza sócio-histórica-cognitiva-cultural baseada na interação, a qual por sua vez, ocorre através do dialogismo. À medida que pesquisas ancoradas nesse paradigma forem desenvolvidas, é possível o advento de subsídios para a elaboração de 
instrumentos diagnósticos, bem como práticas terapêuticas que visam, respectivamente, a antecipar a identificação da DA e a fomentar o desenvolvimento de estratégias interlocutivas para a compensação dos déficits lingüísticos nos sujeitos acometidos pela referida doença.

\section{Referências Bibliográficas}

APRAHAMIAN,I; MARTINELLI, J.E.; YASSUDA, M.S. Doença de Alzheimer: revisão da epidemiologia e diagnóstico. Revista Brasileira de Clínica Médica, $7^{\mathrm{a}}$. Série. São Paulo, p. 7-25, 2009.

AZEVEDO, P.G.; LANDIM, M.E.; FÁVERO, G.P.; CHIAPPETTA, A.L.M.L. Linguagem e Memória na doença de Alzheimer em fase moderada. Revista CEFAC, São Paulo, p.87-198, 2009.

BATISTA, G.F.; NOVAES-PINTO, R.C.; BEILKE, H. Aspectos pragmáticos na linguagem de um sujeito com diagnóstico de demência de Alzheimer. Língua, Literatura e Ensino, Campinas, v. 4, p. 271-281, maio 2009.

BAKHTIN, M. Estética da criação verbal, São Paulo: Martins Fontes, 2000.

BEILKE, H.; NOVAES-PINTO, R.C. Considerações sobre a relação linguagemmemória, à luz da análise linguística dos enunciados de sujeitos com diagnóstico de Demência de Alzheimer. Língua, Literatura e Ensino, Campinas v. 2, p. 65-71, mai. 2007.

BEILKE, H.; NOVAES-PINTO, R.C. A narrativa na demência de Alzheimer: reorganização da linguagem e das "memórias" por meio de práticas dialógicas. Estudos Linguísticos, São Paulo, v. 39, n. 2, p. 557-567, mai.-ago. 2010.

BRANDÃO, L. Perfil discursivo e interativo de pessoas com Doença de Alzheimer. 2005. 186 f. Tese (Doutorado em Psicologia). Instituto de Psicologia da UFRGS, Universidade Federal do Rio Grande do Sul, Porto Alegre, 2005.

BRANDÃO, L.; PARENTE, M. A. M. P.; PEÑA-CASANOVA, J. Turnos e atos de fala do interlocutor de pessoas com doença de Alzheimer. ReVEL, v. 6, n. 11, p. 1-32, ago. 2008 .

BRANDÃO, L.; PARENTE, M.A.M.P; PEÑA-CASANOVA, J. Estratégias Comunicativas de Pessoas com Doença de Alzheimer. Psicologia: Reflexão e Crítica, São Paulo, v.23, n. 2, p. 308-316, 2010.

CARVALHO, I.A.M.; ASSENCIO-FERREIRA, V.J. Análise das habilidades fonológicas no envelhecimento normal e na doença de Alzheimer. Revista CEFAC, São Paulo, v. 4, p. 235-240, 2002. 
CARVALHO, I.A.M. Avaliação funcional das habilidades de comunicação ASHA FACS para população com doença de Alzheimer. 2005. 108 f. Tese (Doutorado em Ciências). Faculdade de Medicina da USP, Universidade de São Paulo, São Paulo, 2005.

CARTHERY, M.T. Caracterização dos Distúrbios da Escrita na Doença de Alzheimer. 2000. 190 f. Dissertação (Mestrado em Neurociências e Comportamento). Faculdade de Neurociências da USP, Universidade de São Paulo, São Paulo, 2000.

CERA, L.M.; ORTIZ, K.Z.; BERTOLUCCI, P.H.F.; MINETT, T.S.C. Manifestações da apraxia de fala na doença de Alzheimer. Revista da Sociedade Brasileira de Fonoaudiologia, v. 16, n. 3, p. 337-343, 2011.

CHOMSKY, N. Estruturas Sintácticas. Lisboa: Edições, 1980.

COUDRY, M. I. H. Neurolingüística Discursiva: Afasia como tradução. In: Maria Irma H. Coudry, Cinthia Ishara e Nirvana Ferraz (orgs.). Estudos da Língua(gem). Número temático: Estudos em Neurolinguística, Vitória da Conquista, v. 6, n.2, p. 6-30, dez. 2008.

CRUZ, F. M. Linguagem, interação e cognição na doença de Alzheimer. 2008. 312 f. Tese (Doutorado em Linguística). Instituto de Estudos da Linguagem da UNICAMP, Universidade Estadual de Campinas, Campinas, 2008.

DAMASCENO, B. P. Envelhecimento cerebral: O problema dos limites entre o normal e o patológico. Arquivos de Neuropsiquiatria, São Paulo, v. 57, n.1, p.78-83, 2009.

FREITAS, M.I.D. Habilidades lingüísticas de pacientes com demência vascular: estudo comparativo com a doença de Alzheimer. 2010. $136 \mathrm{f}$. Tese (Doutorado em Ciências). Faculdade de Medicina da USP, Universidade de São Paulo, São Paulo, 2010.

GOMES, J.B. A linguagem em sujeitos com demência de Alzheimer, sob a ótica de uma concepção enunciativo-discursiva. Estudos Linguísticos, Campinas, v. 36, n. 2, p. 293 300, mai.-ago., 2007.

JAKOBSON, R. Linguística e comunicação. São Paulo: Cultrix, 1956.

LESSA, A.T.M. Tempo em Alzheimer: linguagem,conceito e memória. 2010. $112 \mathrm{f}$. Dissertação (Mestrado em Linguística) Faculdade de Letras da UFRJ, Universidade Federal do Rio de Janeiro, Rio de Janeiro, 2010.

MANSUR, L. L.; CARTHERY, M.T.; CARAMELLI, P.; NITRINI, R. Linguagem e Cognição na Doença de Alzheimer. Psicologia: reflexão e crítica, São Paulo, v. 18, n. 3, p.300-307, 2005.

MARTINS, A.; NOVAES, C. A desintegração do tempo lingüístico em Alzheimer. Veredas on-line Psicolinguística, Juiz de Fora, n. 175-178, 2008. 
MORATO, E. M. O caráter sociocognitivo da metaforicidade: contribuições do estudo do tratamento de expressões formulaicas por pessoas com afasia e com Doença de Alzheimer. Estudos Linguísticos, Belo Horizonte, v. 16, n. 1, p. 157-177, jan.-jun. 2008 .

NOGUCHI, M.S. A Linguagem na doença de Alzheimer: Considerações sobre um modelo de funcionamento Lingüístico. 1997. 102 f. Dissertação (Mestrado em Neurociências). Faculdade de Ciências Médicas da UNICAMP, Universidade Estadual de Campinas, Campinas, 1997.

NOVAES-PINTO, R.C. e BEILKE, H. M. Avaliação de linguagem na demência de Alzheimer. In: Maria Irma H. Coudry, Cinthia Ishara e Nirvana Ferraz (orgs.). Estudos da Língua(gem). Número temático: Estudos em Neurolinguística, Vitória da Conquista. v. 6, n.2, p. 97-126, dez. 2008.

NOVARETTI, T.M.S. Comparação das habilidades de comunicação na depressão de início tardio e doença de Alzheimer. 2009. 149 f. Tese (Doutorado em Medicina). Faculdade de Medicina da USP, Universidade de São Paulo, São Paulo, 2009.

OLIVEIRA, C.C.C. A nomeação de figuras e o acesso lexical na demencia de Alzheimer: um estudo de caso. Distúrbios da Comunicação, São Paulo, v. 17, n.3, p. 359-364, dez. 2005.

ORTIZ, K. Z.; P. H. F. BERTOLUCCI. Alterações de linguagem nas fases iniciais da Demência de Alzheimer. Arquivos de Neuropsiquiatria, São Paulo, v. 63, n. 2, p. 311 317, 2005.

PARENTE, M.A.M.P.; SABOSKINSK, A.P.; FERREIRA, E.; NESPOULOUS, J.L. Memória e compreensão da linguagem no envelhecimento. Estudos Interdisciplinares do Envelhecimento, Porto Alegre, v. 1, p.57-76, 1999.

RINALDI, J. Influência do hipertexto na compreensão textual de pacientes com demência de Alzheimer. 2007. 92 f. Dissertação (Mestrado em Psicologia) - Instituto de Psicologia da UFRGS, Universidade Federal do Rio Grande do Sul, Porto Alegre, 2007.

RINALDI, J.; SILVEIRA, M.; KOCHHANN, R.; PARENTE, M.A.M.P. A compreensão de leitura textual como um instrumento de diagnóstico de pacientes com demência de Alzheimer leve e moderada. Estudos Interdisciplinares do envelhecimento, Porto Alegre, v. 13, n.1, p.171-132, 2008.

RODRIGUES, C. O processamento sintático na demência do tipo Alzheimer. Fórum Linguístico, Florianópolis, v.3, n.1, p. 89-112, jul. 2003. 
SAMARA, A.B. O discurso narrativo na doença de Alzheimer e na demência Fronto-temporal. 2005. 102 f. Tese (Doutorado em Ciências Médicas). Faculdade de Ciências Médicas da UNICAMP, Universidade Estadual de Campinas, Campinas, 2005.

SAMPAIO, N. F. S.; BERNARDO, K. F.; PAIXÃO, T. N. Interação e linguagem: notas sobre a fala de idosos em situações comunicativas. ReVEL, v. 7, n. 13, p. 1-11, 2009.

SAUSSURE, F. Curso de Lingüística Geral. Lisboa: Publicações Dom Quixote, 1986.

SAMPAIO, N.F.S. Alguns aspectos relacionados ao funcionamento da linguagem no envelhecimento. Revista Discursividade. n. 7, dez. 2010.

Artigo recebido em: 10.01.2012

Artigo aprovado em: 04.06.2012 